cellular response to insulin, VSMCs from WKY and SHR were isolated and cultured, and its proteome were comparatively analysed with normal control by 2-DE. Results showed that the proliferation of VSMCs from SHR be more sensitive to insulin stimulation than that VSMCs from WKY. The detectable spots ranged from 537 to 608 on the gels in VSMCs of SHR, and $413 \pm 31$ spots in VSMCs of WKY. The different expressed protein spots in VSMCs of SHR were then isolated and measured by MALDI-TOF-MS. A total of 18 spots showed a sharp clear spectrum, and 13 spots matched with the known proteins from database. These proteins were mainly involved in cytoskeleton, glycometabolism and post-translational processes. Among these proteins, OPN and matrix gla protein were up-regulated expression proteins, whiled $\alpha$-SM actin was downregulated. Furthermore, these preliminarily identified proteins confirmed by RT-PCR and western blotting analysis were coincident with the changes in 2-DE check. In addition, the cytoskeleton changes and migration rate of VSMCs from SHR treated by insulin increased significantly. The results showed that insulin plays a crucial role in activating proliferation and migration of VSMCs, by regulating the phenotype switch of VSMCs.

\section{E0309 THE COMPARISON STUDY OF CORONARY ARTERY LESION IN TYPE 2 DIABETIC PATIENTS WITH CORONARY HEART DISEASE BETWEEN UYGUR AND HAN NATIONALITY}

doi:10.1136/hrt.2010.208967.309

Ren Luo, Chun-Xi Cha, Jian-Ping Yao. Heart Center, The Fourth Affiliated Hospital of Medicine College, Shi-Hezi University, A-Kesu

Objective To study the characteristics of coronary artery lesion between Uygur and Han nationality type 2 diabetic patients with coronary heart disease.

Methods 648 patients were analysed retrospectively in our hospital. The coronary artery lesion was evaluated by the number, location and Gensini scores.

Results Compared with the Han patients, mean age in the Uygur patients was lower in same Gensini scores group $(p<0.05)$. Along with the age increasing, Gensini scores increased gradually. Gensini scores of Uygur patients were significantly higher than those of Han patients in same age groups. The prevalence of three-vessel lesion was the highest in both Han (46.9\%) and Uygur patients (45.9\%). Severity of coronary artery disease increased with age, the prevalence of single-vessel lesion gradually reduced and the prevalence of three- vessel lesion gradually increased.

Conclusions Severity of coronary artery disease increased with age. Severity of coronary artery lesion in Uygur patients is significantly more serious than in Han patients at the same age. Compared with Han patients, the age of onset of similar degree coronary atherosclerosis in the Uygur patients is younger.

\section{e0310 ASYMMETRIC DIMETHYLARGININE AND CAROTID ATHEROSCLEROSIS IN TYPE 2 DIABETES MELLITUS}

doi:10.1136/hrt.2010.208967.310

Xia Wei, Qu Xiufen. Harbin Medical University

Objective Circulating levels of asymmetric dimethylarginine (ADMA), an endogenous nitric oxide synthase inhibitor, are increased in diabetes mellitus (DM). This study was to assess the relationship between plasma ADMA level and carotid atherosclerosis in patients with type $2 \mathrm{DM}$.

Methods A total of 72 newly diagnosed and untreated type 2 DM individuals without manifest cardiovascular disease and 72 healthy controls were studied. Carotid atherosclerosis was determined by ultrasonographically evaluated intima-media thickness (IMT) and plaque score in all subjects. Plasma concentration of ADMA was measured by high-performance liquid chromatography.

Results Fasting blood glucose, haemoglobin A1c, insulin, triglyceride, and ADMA levels, and mean IMT, plaque score were higher in diabetic patients compared with the controls. Univariate and multivariate analyses demonstrated an independent association between ADMA and mean IMT in diabetic patients. On a multiple logistic regression analysis, ADMA was the sole predictor of carotid plaque formation (plaque score $\geq 1.1$ ) (OR 2.43, 95\% CI 1.19 to 4.94, $\mathrm{p}<0.05$ ).

Conclusion Our results suggest that increased levels of ADMA are involved in the development of carotid atherosclerosis in type $2 \mathrm{DM}$.

\section{Epidemiology and Preventive Medicine: Obesity}

e0311 PREVALENCE OF CARDIOVASCULAR DISEASE BIOLOGICAL RISK FACTOR CLUSTERING AMONG OVERWEIGHT AND OBESE POPULATION IN BEIJING COMMUNITY-RESULTS FROM CCEIP

doi:10.1136/hrt.2010.208967.311

${ }^{1} \mathrm{Fu}$ Yuanyuan, ${ }^{2} \mathrm{Yu}$ Jinming, ${ }^{3}$ Wang Jiahong, ${ }^{3}$ Sun Yihong, ${ }^{4}$ Liu Huifang, ${ }^{3} \mathrm{Hu}$ Dayi ${ }^{1}$ Medical School of Tongii University; ${ }^{2}$ Fudan University School of Public Health; ${ }^{3}$ Peking University People's Hospital; ${ }^{4}$ Beijing Daxing Lucheng Health Center

Objective To investigate prevalence of CVD biological risk factor clustering among overweight and obese residents in Beijing community.

Method Cross-sectional data of 9786 subjects from CCEIP were obtained for analysis. Participants were divided into 3 groups (Normal, overweight and obese population) based on body mass index (BMI). Body examinations were done to record blood pressure. Overnight fasting plasma samples were drawn to determinate blood lipid and glucose levels.

Results 1) $10.5 \%, 22.5 \%$ and $37.9 \%$ subjects from normal, overweight and obese group had $\geq 2$ biological CVD risk factors. $(11.5 \%$, $21.7 \%, 51.5 \%$ in men and $9.5 \%, 17.2 \%, 24.4 \%$ in women). The proportion of biological risk factor clustering patients elevated with the increase of BMI. Clustering hazard will increase by $21 \%$ when BMI increase every 1 unit. Clustering prevalence was higher in overweight and obese men than women (both $p<0.001)$. 2) Prevalence of clustering increased with ageing in population. However there was no significant difference among youth, middle aged and elderly obese male population $(49.2 \%, 49.7 \%$ and $56.1 \%$, $=2.52$ $\mathrm{p}=0.285$ ). 3) The most common clinical symptoms complex of clustering was hypertension and dyslipidemia.

Conclusion The prevalence of CVD risk factor clustering was relatively high in overweight and obese population. Strengthen intervention should be taken in obese population, especially the young men, to prevent CVD events.

\section{e0312 EVALUATION ON THE DIAGNOSTIC VALUE OF BODY MASS INDEX IN PREDICTING OBSTRUCTIVE SLEEP APNEAHYPOPNEA SYNDROME IN CHINESE ADULTS}

doi:10.1136/hrt.2010.208967.312

Zeng Jin, GU Yalan, MA Furong. The Third Hospital of Peking University

Objective To evaluate diagnostic value of body mass index (BMI) in screening and diagnosing obstructive sleep apnoea-hypopnoea syndrome and to determine the reference standard of body mass index (BMI) in both male and female population by receiver operating characteristic (ROC) curve. 
Method A total of 1348 subjects (1063 male and 285 female) who experienced polysomnography (PSG) during 2004-2010 in The Third Hospital of Peking University ENT department were included. The correlativity between apnoea-hypopnoea index (AHI) and BMI was conducted by pearson correlation test. The diagnostic value and reference standard in both male and female were analysed by ROC curve.

Result 1) A positive relationship between AHI and BMI was detected, both in male and female subjects, with pearson coefficients 0.423 and 0.419 ( $p<0.001$ ). 2) Results of ROC analysis show a favourable value of BMI in diagnosing OSHAS, especially in male subjects. In male population, area under curve (AUC) was 0.759 (95\% CI 0.724 to $0.795)$ with a best cutoff value $25.3 \mathrm{~kg} / \mathrm{m}^{2}$. The sensitivity and specificity in man were $74 \%$ and $61 \%$, respectively; in women, area under curve (AUC) was 0.691 (95\% CI 0.632 to 0.751 ) with a best cutoff value $24.7 \mathrm{~kg} / \mathrm{m}^{2}$. The sensitivity and specificity were $68 \%$ and $61 \%$. The screening cutoff values were $22.4 \mathrm{~kg} / \mathrm{m}^{2}$ and $20.7 \mathrm{~kg} / \mathrm{m}^{2}$ in male and female, with a sensitivity of $95 \%$. The diagnostic cutoff values were $30.0 \mathrm{~kg} / \mathrm{m}^{2}$ and $36.0 \mathrm{~kg} / \mathrm{m}^{2}$, with a specificity of $95 \%$.

Conclusion BMI seemed a better diagnostic value of OSAHS in diagnosing than screening, especially in male population. Sexualseparated cutoff values should be use in clinical practice to enhance diagnostic accuracy.

\section{e0313 THE DETECTION OF PLASMA VISFATIN IN OBESE PATIENTS WITH CORONARY ARTERY DISEASE}

doi:10.1136/hrt.2010.208967.313

Yu Qin, Hong-Jiu Yu, Tian Lv. Department of the First Hospital of Dalian Medical University

Objective To explore the level of plasma visfatin in patients with coronary artery disease (CAD) and different BMI, and evaluate the relationship between plasma visfatin and coronary lesion severity in obese patients with CAD

Methods 59 causes were undergone coronary angiography and divided into three sub-groups: normal weight (13 causes) $\left(\mathrm{BMI}<24 \mathrm{~kg} / \mathrm{m}^{2}\right)$, over weight (31 causes) $\left(24 \leq \mathrm{BMI}<28 \mathrm{~kg} / \mathrm{m}^{2}\right)$ and obese groups (15 causes) ( $\left.\mathrm{BMI} \geq 28 \mathrm{~kg} / \mathrm{m}^{2}\right)$. The levels of plasma visfatin of $\mathrm{CAD}$ group and control group were measured and compared. The correlation between plasma visfatin and coronary lesion severity score with different BMI was analysed.

Result The plasma visfatin level was higher in the CAD group $(547.21 \pm 94.18 \mathrm{ng} / \mathrm{ml})$ comparing with the control group $(470.86 \pm 87.99 \mathrm{ng} / \mathrm{ml})$. In obese patients with CAD the plasma visfatin level $(586.62 \pm 96.77 \mathrm{ng} / \mathrm{ml})$ increased more significantly and it was positively correlated with Gensini score $(p<0.05)$.

Conclusion The level of plasma visfatin is closely correlated with coronary lesion severity in obese patients with $\mathrm{CAD}$. It might be used as a new clinical marker to predict the severity of coronary lesion in obese patients with $\mathrm{CAD}$.

\section{e0314 ASSOCIATION OF OBESITY WITH RENIN-ANGIOTENSIN SYSTEM}

doi:10.1136/hrt.2010.208967.314

Ali Raza Kazmi, Muhammad Ismail, Mansoor Qaiser. Ibgeg-9/1 Islamabad

Overweight and obesity are highly associated with multiple comorbidities, elevated blood pressure values, dyslipidaemia, reduced insulin sensitivity and alterations of large and minor vessels. The essential role of the renin-angiotensin system (RAS) in controlling blood pressure has well been established. Genes encoding components of the RAS have been proposed as candidate genes that determine genetic predisposition to hypertension and the risk of developing cardiovascular complications. The role of these genes in obesity is not well understood. Several studies have showed their association with obesity in animal models.

\section{Clinical and Research Medicine: Cardiovas- cular Clinical Pharmaceutical Research e0315 EXPLORE THE RELATIONSHIP BETWEEN LEFT VENTRICULAR MURAL THROMBUS AND LEFT VENTRICULAR ANEURYSM AFTER ACUTE MYOCARDIAL INFARCTION}

doi:10.1136/hrt.2010.208967.315

Zhang Zhiping, Cheng Guohong, Su Xi. Department of Cardiology, Wuhan Asia Heart Hospital, Wuhan 430022, China

Objective To explore the relationship between left ventricular mural thrombus (LVMT) and left ventricular aneurysm (LVA) after acute myocardial infarction (AMI).

Methods The clinical materials of 66 consecutive patients with LVA after AMI were retrospectively analysed. These patients were divided into two groups according the result of LVMT formation diagnosed by echocardiographic. 30 cases with LVMT entered group LVMT and the other 36 cases entered group without LVMT. The mean left atrial (LA), LV end-diastolic and end-systolic diameter, thickness of the interventricular septum (IVS) and LV posterior wall (PW), and the size of the aneurysm as well as the LV ejection fraction (LVEF) were compared among two groups.

Results According to results of analysis, the mean LA, LV enddiastolic and end-systolic diameter, PW thickness, and the size of the aneurysm did not differ between patients with and without LVMT. The thickness of the IVS were greater in patients in with LVMT than in those without LVMT $(1.17 \pm 0.26$ vs $1.04 \pm 0.19 \mathrm{~cm}$, respectively; $\mathrm{p}=0.02$ ).

Conclusions Left ventricular aneurysm formation after AMI could be related to LVMT. However, the size of the aneurysm was not significant associated with LVMT.

\section{e0316 THE scD40L AND CIRCULATION ENDOTHELIAL PROGENITOR CELLS CHANGE OF DANHONG COMBINED NAOXINTONG THERAPY TO ACUTE CORONARY SYNDROME PATIENTS WITH PERCAUTIOUS CORONARY INTERVENTION}

doi:10.1136/hrt.2010.208967.316

${ }^{1}$ Lu Jing-qian, ${ }^{2}$ Li Yi, ' Lv Yun, ${ }^{1}$ Pan Ya-ping, ' ${ }^{1}$ Gao Yan, ${ }^{1}$ Yang Feng. ${ }^{1}$ Department of Cardiologythe 3th Hospital of Yunnan Province; ${ }^{2}$ Saints Heart Hospital of Yunnan

Objective To observe the effects of Danhong injection combined naoxintong pills with acute coronary syndrome (ACS) patients who accepted percautious coronary intervention (PCI) about recelently outcome and changes of blood serum soluble CD40 ligand (sCD40L) and Circulation Endothelial Progenitor Cells (EPCs).

Methods 91 ACS patients with PCI therapy were randomly divided into Buchang group $(n=48)$ and normaltherapy group $(n=43)$, after PCI, normaltherapy group were given coronary heart disease second prevention drugs, and Buchang group were added danghong and naoxintong at the basis of coronary heart disease second prevention drugs. the outcome after PCI 3 months, 6 months and changes of sCD40L and EPCs were observed.

Results In two groups, cardiac death, myocardial infarction, stroke, angina, re-hospitality were no significantly difference $(p>0.05)$ but the left ventricular ejection fraction were more higher in Buchang group than normaltherapy group $(61.5 \%$ vs $55.3 \%, \mathrm{p}<0.05)$. 6 miniters walk distence test were more higher in Buchang group 